\title{
AFRONTAMIENTO DEL ESTRÉS EN ADOLESCENTES ESTUDIANTES DE NIVEL MEDIO SUPERIOR
}

\section{STRESS COPING SKILLS IN ADOLESCENT HIGH SCHOOL STUDENTS}

\author{
Brenda Guadalupe Morales Salinas* y Alejandra Moysén Chimal** \\ Universidad Autónoma del Estado de México, México
}

\section{RESUMEN}

La adolescencia supone una reorganización de las interacciones sociales, interpersonales (Ávila, Heredia, Gómezy Martínez, 2006; Cardozo y Alderete 2009), lo que genera estrés (Frydenberg y Lewis, 2000). Para afrontarla, se emplea un conjunto de acciones y cogniciones que capacitan para tolerar o minimizar los efectos producidos (González, Montoya, Martina y Bernabéu, 2002). El propósito de la investigación es describir las estrategias de afrontamiento que emplean los adolescentes estudiantes del nivel medio superior, realizando comparaciones por género y edad. Se trabajó con 724 adolescentes, 413 mujeres y 311 hombres, con un rango de edad entre 14 a 17 años, del Valle de Toluca, Estado de México. Se aplicó la Escala de Afrontamiento para Adolescentes (ACS), elaborada por Frydenberg y Lewis (2000). Los resultados obtenidos de las estrategias que emplean los adolescentes ante situaciones estresantes son: esforzarse y tener éxito, buscar diversiones relajantes y preocuparse. Las mujeres emplean más estrategias centradas en resolver el problema e intervienen sus emociones, a diferencia de los hombres que evaden el problema y son más reservados emocionalmente. Con respecto a la comparación por edad, se encontró que los de 16 años emplean estrategias de evasión del problema. Se discuten las implicancias del estudio.

Palabras clave: adolescentes, estrés, afrontamiento, género, edad.

\section{ABSTRACT}

Adolescence entails a reorganization of social, interpersonal interactions (Ávila, Heredia, Gómez \& Martínez, 2006; Cardozo \& Alderete, 2008), which generates stress (Frydenberg \& Lewis, 2009). To deal with it, a variety of actions and cognitions are used, which prepare us to tolerate or minimize the effects produced by it (González, Montoya, Martina and Bernabeu, 2002). The purpose of this research is to describe the coping strategies used by adolescent high school students, making comparisons by sex and age. The work was done with 724 adolescents: 413 females and 311 males; ranging between 14 and 17 years of age, from Valle de Toluca, Estado 
de México. The Adolescent Coping Scale (ACS), developed by Frydenberg and Lewis (2000) was applied. The results obtained regarding the strategies used by adolescents in stressful situations are: to work hard and achieve, seek relaxing diversions, and worry. Females use more strategies focused on solving the problem, and they handle their emotions, unlike males who evade the problem and are more emotionally reserved. Regarding the age comparison, it was found that 16-year olds employ problem-evasion strategies. The implications of this study are discussed.

Keywords: adolescents, stress, coping, sex, age.

\section{INTRODUCCIÓN}

La adolescencia es considerada como una etapa de adaptación, crisis, estrés y desajuste; es un periodo que demanda de la persona todo su esfuerzo para adaptarse a nuevas necesidades personales y sociales (Enríquez, Lira, Balcázar, Bonilla y Gurrola, 2009).

Es un período de profundos cambios, el cual supone una reorganización de las interacciones sociales, ya que hay modificaciones en las relaciones interpersonales con pares, padres y pareja (Ávila et al., 2006; Cardozo y Alderete 2009). Así, el adolescente se ve obligado a cumplir ciertos papeles sociales en relación a sus compañeros, a los miembros del otro sexo $\mathrm{y}$, al mismo tiempo, a conseguir buenos resultados escolares y a tomar decisiones sobre su carrera profesional, lo que le puede generar estrés (Frydenberg y Lewis, 2000).

El estrés es un estímulo considerado como amenazante, que provoca una reorganización en la vida de la persona, dependiendo de la intensidad con que se presente y el grado en que sea percibido (Buendía, 1993; Casullo, 1998; Lazarus y Folkman, 1986; Oblitas y Becoña, 2000). Con respecto al estrés psicosocial, es una relación entre el individuo y el entorno, que es evaluado como amenazante y puede poner en peligro el bienestar de la persona; lo cual desencadena cambios en la rutina de vida del individuo, provocando nuevas condiciones y necesidades a las cuales tiene que responder (Lazarus y Folkman, 1986).

Tales experiencias estresantes afectan cada vez más al adolescente, interfiriendo en su comportamiento y salud. La mayor parte de las repercusiones indeseables, que aquejan a los adolescentes sometidos a estrés, son de carácter psicológico, tales como ansiedad, depresión y problemas de aprendizaje (Gaeta y Martín, 2009). Esto, debido a los efectos que tiene el estrés en el funcionamiento fisiológico e inmunológico ante la acumulación de los eventos estresantes, lo cual puede afectar el bienestar y la salud del adolescente (Barra, Cerna, Kramm y Véliz, 2006).

Estas condiciones se aplican a eventos estresantes, significativos o intensos, y, además, a aquellos estresores menores o problemas cotidianos que pueden llegar a tener un efecto acumulativo y aumentar la vulnerabilidad al presentar problemas de salud física o mental (Barra et al., 2006). No obstante, las potenciales consecuencias del estrés están reguladas por el afrontamiento, es decir, por 
un conjunto de acciones y cogniciones que capacitan al individuo adolescente para tolerar, evitar o minimizar los efectos producidos por un evento estresante (González et al., 2002).

Es, entonces, posible, utilizar estrategias de afrontamiento positivas, las cuales reducen el estrés y promueven la salud a largo plazo, contrarias a las inadaptativas, que reducen el estrés únicamente a corto plazo, provocando un efecto nocivo en la salud si transcurre más tiempo. Al responder a este tipo de situaciones, se puede generar comportamientos menos adaptativos y mayor vulnerabilidad en el adolescente, afectando su desarrollo psicológico e incluso su vida. Tales estrategias se desarrollan por las experiencias de la niñez y se modifican de acuerdo a los resultados obtenidos con relación a las situaciones estresantes (Veloso, Caqueo, Caqueo, Muñoz y Villegas, 2010).

Esta capacidad de afrontamiento, es decir, las estrategias conductuales y cognitivas que emplea el adolescente, le sirve para lograr una adaptación y una transición efectiva, repercutiendo no sólo en su vida, sino también en la de su familia y en el aspecto social. La forma en que el adolescente afronta sus problemas, puede establecer patrones de conducta para su vida adulta (Frydenberg y Lewis, 2000).

González et al. (2002), sugieren que existen similitudes en mujeres y hombres con respecto al empleo de estrategias de afrontamiento y a la utilización de un estilo focalizado en la emoción. Sin embargo, las mujeres tienden a emplear con mayor frecuencia la búsqueda de apoyo social, por lo que utilizan estilos de afrontamiento de relación con los demás, así como se fijan en lo positivo y se concentran en la resolución de problemas. Asimismo, se ha encontrado que las mujeres emplean un estilo de afrontamiento inadaptado, lo cual se ve reflejado a partir de autoinculparse y hacerse ilusiones para sentirse mejor y relajar la tensión.

En contraparte, los varones se centran en mejorar sus logros académicos y mantener su bienestar psicológico, utilizando más la distracción física, ignorando el problema o reservándolo para sí mismos. Gaeta y Martín (2009) mencionan que, con respecto al género, el proceso de afrontamiento puede estar influido por la cultura y contexto.

Por otra parte, en un estudio reportado por Moysén (2011), se encontró que los alumnos de nivel medio superior emplean diversas estrategias para hacer frente a los sucesos de vida estresantes, entre las que se encuentran el apoyo social, la planeación y el afrontamiento activo; ésta última consiste en hacer planes específicos para manejar el estrés y llevarlos a cabo.

Con respecto a las estrategias de afrontamiento en los adolescentes y la edad, González et al. (2002) refieren que los de 15 a 18 años poseen un repertorio de respuestas de afrontamiento más amplio, comparados con los de menor edad, quienes desarrollan una mayor variedad de estrategias de afrontamiento y usan métodos que reducen el impacto del estrés de manera directa, involucrando el componente cognitivo (resolución de problemas planificados, reapreciación positiva y autocontrol), esto debido al surgimiento del pensamiento de las operaciones formales, que ayuda a los adolescentes a elegir estrategias de afrontamiento efectivas. Estos son capaces de pensar de manera abstracta, al considerar varios puntos de vista y al evaluar las posibles consecuencias (Gaeta y Martín, 2009).

No obstante, el afrontamiento de los adolescentes ante el estrés se puede presentar en función de varios factores, tales como los recursos 
y habilidades sociales del individuo para resolver problemas, así como las creencias, los valores, las limitaciones personales, ambientales y el grado de amenaza que los adolescentes perciban (Gaeta y Martín, 2009).

Por lo tanto, las estrategias de afrontamiento utilizadas por los adolescentes se presentan dependiendo de las experiencias y el contexto en el que se desenvuelvan, ya que el papel que desempeñan en las diferentes esferas de su vida, desde sus relaciones familiares, escolares, de pareja, entre otras, influye en el desarrollo de estrategias para reducir el estrés, sean éstas positivas o inadaptativas. Asimismo, existen diferencias con respecto a la presencia de las estrategias de afrontamiento en relación al sexo del individuo, viéndose afectados varios aspectos de su vida, por lo que la presente investigación se enfoca en identificar las estrategias de afrontamiento empleadas por adolescentes estudiantes del nivel medio superior, considerando el género y la edad. Todo ello deriva en las siguientes preguntas, que guiaron la presente investigación:

1. ¿Cuáles son las estrategias de afrontamiento, ante situaciones estresantes, más frecuentemente empleadas por los adolescentes, estudiantes del nivel medio superior?
2. ¿Qué diferencias existen en las estrategias de afrontamiento empleadas ante situaciones estresantes, en estudiantes de nivel medio superior, según género y edad?

\section{MÉTODO}

\section{Diseño de investigación}

Se llevó a cabo un estudio de corte cuantitativo a través de un diseño no experimental, transversal descriptivo, el cual se realiza sin manipular deliberadamente variables; recolecta datos en un sólo momento, en un tiempo único. Su propósito es describir variables y analizar su incidencia e interrelación en un momento dado (Hernández, Fernández y Baptista, 2010).

\section{Participantes}

Se realizó un muestreo no probabilístico intencional, el cual consiste en que la elección de los elementos no depende de la probabilidad sino de las causas relacionadas con las características de la investigación, se eligen los individuos que se estima que son representativos o típicos de la población que puedan facilitar la información (Hernández, et al., 2010). La muestra se conformó por 724 adolescentes (413 mujeres y 311 hombres), con un rango de edad de 14 a 17 años, que realizan sus estudios de nivel medio superior en cuatro

Tabla 1

Frecuencia y porcentajes de participantes, de acuerdo a la edad y el género

\begin{tabular}{|c|c|c|c|c|}
\hline \multirow[t]{3}{*}{ Edad } & \multicolumn{4}{|c|}{ Género } \\
\hline & \multicolumn{2}{|c|}{ Masculino } & \multicolumn{2}{|c|}{ Femenino } \\
\hline & F & $\%$ & $\mathrm{~F}$ & $\%$ \\
\hline 14 años & 14 & 1.93 & 23 & 3.17 \\
\hline 15 años & 160 & 22.09 & 211 & 29.14 \\
\hline 16 años & 69 & 9.53 & 103 & 14.22 \\
\hline 17 años & 68 & 9.39 & 76 & 10.49 \\
\hline Total & 311 & 42.94 & 413 & 57.02 \\
\hline
\end{tabular}


escuelas públicas del Valle de Toluca, Estado de México (ver tabla 1).

\section{Instrumento}

Se empleó la Escala de Afrontamiento para Adolescentes (ASC, Frydenberg y Lewis, 2000).

Esta escala permite examinar las conductas de afrontamiento de los adolescentes, al obtener información sobre cómo actúan en diversas situaciones estresantes. Está compuesta por 80 ítems, 79 de tipo cerrado y uno abierto, que permiten evaluar con fiabilidad las 18 estrategias de afrontamiento propuestas en la literatura: buscar apoyo social, concentrarse en resolver el problema, esforzarse y tener éxito, preocuparse, invertir en amigos íntimos, buscar pertenencia, hacerse ilusiones, falta de afrontamiento o no afrontamiento, reducción de la tensión, acción social, ignorar el problema, autoinculparse, reservarlo para sí, buscar apoyo espiritual, fijarse en lo positivo, buscar ayuda profesional, buscar diversiones relajantes y distracción física.

Los 79 elementos se califican mediante una escala de cinco puntos, que van de Nunca lo hago a Lo hago con mucha frecuencia; con un índice de confiabilidad interna Alfa de Cronbach $\alpha=.90$, en la adaptación mexicana hecha por Bárcenas y Martínez (2008), y un porcentaje de varianza explicada de 57.498\%. Las escalas ACS han sido empleadas en estudios realizados en México, demostrando índices de validez y confiabilidad aceptables (Bárcenas y Martínez, 2008; Contreras, Chávez y Aragón, 2012; Martínez y Pedroza, 2011).

\section{Procedimiento}

Se obtuvo la autorización de los directivos, de las instituciones educativas, de nivel medio superior, en las que se llevó a cabo la investigación para la aplicación del instrumento. Los docentes encargados de la clase, en el momento de la aplicación de la prueba, fueron los que invitaron a los alumnos a participar de manera voluntaria. Cada uno de los participantes firmó el consentimiento informado; asimismo, se les indicó que los instrumentos se analizarían de manera grupal. Las aplicaciones duraron un promedio de 15 minutos. El análisis de los datos se procesó utilizando el paquete estadístico SPSS versión 20, calculando media, desviación estándar, t student y análisis de varianza de una sola vía. Se trabajó con una prueba de hipótesis de $\mathrm{p} \leq 0.05$.

\section{RESULTADOS}

Los resultados se organizan de acuerdo a los objetivos presentados, es decir, se empieza por la descripción de las estrategias de afrontamiento que emplean los adolescentes, luego se realiza la comparación por género y finalmente la comparación por edad.

La tabla 2 muestra las estrategias de afrontamiento ante situaciones estresantes, que emplean los adolescentes estudiantes del nivel medio superior. De acuerdo con los resultados obtenidos, las estrategias de esforzarse y tener éxito ( $M=73.005)$, buscar diversiones relajantes $(M=72.136)$ y preocuparse $(M=70.812)$ son las que utilizan con mayor frecuencia.

La tabla 3 muestra los resultados de la comparación de las estrategias de afrontamiento por género, a partir de la prueba $t$ student. Los datos obtenidos muestran diferencia estadísticamente significativa en 12 estrategias, de las 18 que conforman el instrumento. Las participantes del género femenino utilizan con mayor frecuencia las siguientes: buscar apoyo social, esforzarse y tener éxito, preocuparse, buscar pertenencia, hacerse ilusiones, reducción de la tensión, autoinculparse, buscar apoyo espiritual y fijarse en lo positivo; en contraparte, el género masculino presenta una media más alta en ignorar el problema, buscar diversiones 


\section{Tabla 2}

Estrategias de afrontamiento de los adolescentes estudiantes del nivel medio superior del Valle de Toluca

\begin{tabular}{|c|c|c|}
\hline Estrategias & M & $\mathrm{DE}$ \\
\hline 1. Esforzarse y tener éxito & 73.005 & 13.877 \\
\hline 2. Buscar diversiones relajantes & 72.136 & 16.302 \\
\hline 3. Preocuparse & 70.812 & 14.671 \\
\hline 4. Concentrarse en resolver el problema & 68.342 & 15.389 \\
\hline 5. Fijarse en lo positivo & 68.038 & 15.838 \\
\hline 6. Distracción física & 67.756 & 21.308 \\
\hline 7. Buscar pertenencia & 64.132 & 14.268 \\
\hline 8. Invertir en amigos íntimos & 62.701 & 16.103 \\
\hline 9. Buscar apoyo social & 61.066 & 16.246 \\
\hline 10. Buscar ayuda profesional & 56.933 & 19.039 \\
\hline 11. Hacerse ilusiones & 54.547 & 14.535 \\
\hline 12. Reservarlo para sí & 51.049 & 17.511 \\
\hline 13. Autoinculparse & 47.948 & 17.155 \\
\hline 14. Buscar apoyo espiritual & 46.291 & 15.585 \\
\hline 15. Reducción de la tensión & 37.917 & 13.234 \\
\hline 16. Falta de afrontamiento o no afrontamiento & 36.707 & 12.573 \\
\hline 17. Ignorar el problema & 35.697 & 13.259 \\
\hline 18. Acción social & 35.642 & 11.729 \\
\hline
\end{tabular}

Nota: Valores de frecuencia en que se utilizan las estrategias: de 90-100 se utilizan con mucha frecuencia, 70-89 a menudo, de 50-69 algunas veces, de 30-49 raras veces y de 20-29 estrategia no utilizada. 
Tabla 3

Comparación de las estrategias de afrontamiento en relación al género, en estudiantes de nivel medio superior del Valle de Toluca

\begin{tabular}{|c|c|c|c|c|c|c|}
\hline \multirow[t]{2}{*}{ Estrategias } & \multicolumn{2}{|c|}{ Femenino } & \multicolumn{2}{|c|}{ Masculino } & \multirow[t]{2}{*}{$\mathrm{t}$} & \multirow[t]{2}{*}{ Sig. } \\
\hline & M & $\mathrm{DE}$ & M & $\mathrm{DE}$ & & \\
\hline Buscar apoyo social & 62.67 & 16.26 & 58.93 & 15.99 & 3.085 & .002 \\
\hline Concentrarse en resolver el problema & 68.71 & 15.79 & 67.84 & 14.85 & .760 & .447 \\
\hline Esforzarse y tener éxito & 74.21 & 13.88 & 71.39 & 13.72 & 2.721 & .007 \\
\hline Preocuparse & 72.50 & 14.81 & 68.56 & 14.19 & 3.604 & .000 \\
\hline Invertir en amigos íntimos & 62.69 & 16.20 & 62.71 & 15.99 & .018 & .986 \\
\hline Buscar pertenencia & 65.04 & 14.62 & 62.91 & 13.71 & 2.007 & .045 \\
\hline Hacerse ilusiones & 55.86 & 14.61 & 52.79 & 14.25 & 2.824 & .005 \\
\hline Falta de afrontamiento o no afrontamiento & 37.14 & 12.54 & 36.12 & 12.61 & 1.074 & .283 \\
\hline Reducción de la tensión & 40.25 & 13.18 & 34.81 & 12.67 & 5.583 & .000 \\
\hline Acción social & 35.29 & 11.42 & 36.10 & 12.12 & .930 & .353 \\
\hline Ignorar el problema & 34.70 & 12.46 & 37.00 & 14.16 & 2.317 & .021 \\
\hline Autoinculparse & 49.15 & 17.73 & 46.35 & 16.23 & 2.209 & .028 \\
\hline Reservarlo para sí & 51.54 & 17.68 & 50.38 & 17.28 & .888 & .375 \\
\hline Buscar apoyo espiritual & 47.66 & 15.83 & 44.46 & 15.08 & 2.742 & .006 \\
\hline Fijarse en lo positivo & 69.98 & 15.28 & 65.45 & 16.21 & 3.820 & .000 \\
\hline Buscar ayuda profesional & 56.75 & 19.11 & 57.17 & 18.96 & .290 & .772 \\
\hline Buscar diversiones relajantes & 71.06 & 16.17 & 73.55 & 16.39 & 2.034 & .042 \\
\hline Distracción física & 64.18 & 21.19 & 72.49 & 20.55 & 5.292 & .000 \\
\hline
\end{tabular}




\section{Tabla 4}

Comparación de las estrategias de afrontamiento en relación a la edad, en estudiantes de nivel medio superior del Valle de Toluca

\begin{tabular}{|c|c|c|c|c|c|c|c|c|c|c|}
\hline \multirow[t]{2}{*}{ Estrategias } & \multicolumn{2}{|c|}{14} & \multicolumn{2}{|c|}{15} & \multicolumn{2}{|c|}{16} & \multicolumn{2}{|c|}{17} & \multirow[t]{2}{*}{$\mathrm{F}$} & \multirow[t]{2}{*}{ Sig. } \\
\hline & $\mathrm{M}$ & $\mathrm{DE}$ & M & DE & M & $\mathrm{DE}$ & M & $\mathrm{DE}$ & & \\
\hline Buscar apoyo social & 58.05 & 14.31 & 61.74 & 16.45 & 60.67 & 15.57 & 60.5 & 16.98 & .721 & .540 \\
\hline $\begin{array}{l}\text { Concentrarse en resolver } \\
\text { el problema }\end{array}$ & 68.00 & 17.07 & 68.23 & 15.99 & 68.13 & 14.44 & 68.9 & 14.55 & .095 & .963 \\
\hline Esforzarse y tener éxito & 72.54 & 15.08 & 73.66 & 14.18 & 72.51 & 13.89 & 72.0 & 12.74 & .599 & .616 \\
\hline Preocuparse & 67.13 & 15.29 & 69.82 & 15.13 & 72.69 & 13.75 & 72.05 & 14.08 & $2.64 *$ & .048 \\
\hline $\begin{array}{l}\text { Invertir en amigos } \\
\text { íntimos }\end{array}$ & 60.00 & 16.46 & 62.93 & 16.94 & 63.58 & 14.72 & 61.75 & 15.38 & .710 & .546 \\
\hline Buscar pertenencia & 62.59 & 15.00 & 65.09 & 14.31 & 63.88 & 13.71 & 62.3 & 14.51 & 1.49 & .215 \\
\hline Hacerse ilusiones & 53.51 & 14.55 & 54.61 & 14.57 & 54.41 & 13.88 & 54.77 & 15.30 & .082 & .970 \\
\hline $\begin{array}{l}\text { Falta de afrontamiento o } \\
\text { no afrontamiento }\end{array}$ & 31.89 & 10.81 & 35.71 & 11.83 & 39.20 & 14.06 & 37.50 & 12.42 & $5.11 *$ & .002 \\
\hline Reducción de la tensión & 35.45 & 13.66 & 35.95 & 12.18 & 41.02 & 14.03 & 39.88 & 13.83 & $7.56^{*}$ & .000 \\
\hline Acción social & 35.94 & 10.79 & 34.74 & 11.02 & 37.44 & 12.87 & 35.72 & 12.16 & 2.09 & .100 \\
\hline Ignorar el problema & 30.94 & 9.98 & 35.09 & 12.87 & 37.70 & 13.59 & 36.07 & 14.21 & $3.22 *$ & .022 \\
\hline Autoinculparse & 45.00 & 16.99 & 46.45 & 16.63 & 50.81 & 17.83 & 49.13 & 17.30 & 3.15 & .024 \\
\hline Reservarlo para sí & 47.97 & 16.89 & 50.17 & 17.17 & 52.26 & 16.57 & 52.63 & 19.44 & 1.36 & .253 \\
\hline Buscar apoyo espiritual & 49.05 & 14.32 & 46.13 & 15.82 & 46.10 & 14.64 & 46.21 & 16.43 & .409 & .747 \\
\hline Fijarse en lo positivo & 69.32 & 16.54 & 69.46 & 15.98 & 66.27 & 15.31 & 66.14 & 15.65 & 2.48 & .059 \\
\hline Buscar ayuda profesional & 56.48 & 20.87 & 57.72 & 19.60 & 55.72 & 16.91 & 56.4 & 19.55 & .478 & .697 \\
\hline $\begin{array}{l}\text { Buscar diversiones } \\
\text { relajantes }\end{array}$ & 66.02 & 16.86 & 71.75 & 15.91 & 74.23 & 16.26 & 72.18 & 16.87 & $2.76^{*}$ & .041 \\
\hline Distracción física & 65.83 & 18.70 & 69.01 & 21.50 & 68.90 & 20.50 & 63.6 & 22.01 & 2.51 & .057 \\
\hline
\end{tabular}


relajantes y distracción física, por lo que tienden a emplear con mayor frecuencia estas estrategias.

La tabla 4 muestra los resultados obtenidos del análisis de varianza, de una sola vía, de la comparación entre las estrategias de afrontamiento ante situaciones estresantes y la edad de adolescentes estudiantes de nivel medio superior del Valle de Toluca. Los datos obtenidos muestran diferencias estadísticamente significativas para las siguientes estrategias: preocuparse, falta de afrontamiento o no afrontamiento, reducción de la tensión, ignorar el problema, autoinculparse y buscar diversiones relajantes. Los estudiantes de 16 años son los que presentan la media más alta, por lo que emplean con mayor frecuencia estas estrategias.

\section{DISCUSIÓN}

De acuerdo con los resultados obtenidos, las estrategias afrontamiento ante situaciones estresantes, empleadas por adolescentes estudiantes de nivel medio superior del Valle de Toluca, son: esforzarse y tener éxito, buscar diversiones relajantes y preocuparse, por lo que tienden a afrontar situaciones estresantes empleando más un estilo de resolución de problemas, es decir, ante un acontecimiento que les ocurre llevan a cabo esfuerzos dirigidos a modificar los eventos ambientales causantes del estrés, intentando solucionar el problema o al menos disminuir su impacto (Lazarus y Folkman, 1986).

Wade y Tavris (2003) y Gaeta y Martín (2009) refieren que el afrontamiento no es la estrategia única que se aplica a todas las situaciones, las personas afrontan de formas diferentes, lo cual está en función de los recursos y habilidades sociales del individuo para resolver problemas, así como las creencias, valores, limitaciones personales, ambientales y del grado de amenaza percibido; las personas pueden utilizar varios estilos y estrategias; les ayuda a sobrevivir al estrés, pero también les apoya a resistir a la adversidad y a progresar, aprendiendo de sus experiencias.

Los adolescentes del presente estudio, afrontan los sucesos estresantes de forma diferente, es decir, emplean sus habilidades para hacer frente a los acontecimientos de su vida; una manera de cómo manejan el estrés tiene que ver con esforzarse y tener éxito, la cual consiste en manifestar conductas de trabajo e implicación personal como hacer tareas, asistir a clases y triunfar en lo que se hace; asimismo, emplean estrategias dirigidas a buscar diversiones relajantes, como situaciones de ocio (oír música, leer un libro, ver televisión, salir a divertirse), y la estrategia de preocuparse, que se caracteriza por elementos de la felicidad futura del adolescente.

Solís y Vidal (2006) mencionan que el comportamiento del adolescente se encuentra inscrito dentro de un conjunto de tareas, como el logro de una identidad, incorporación de valores, independencia económica, social y emocional de los padres, perspectivas de futuro y un desarrollo psicosexual. Sin embargo, las tareas descritas podrían convertirse en fuentes de estrés con problemas vinculados a la escuela, incertidumbre respecto al futuro, dificultades familiares, presión social, problemas relacionados al uso del tiempo libre, a las relaciones amorosas y contrariedades en relación a sí mismos, por lo que el lograr desarrollar estas tareas supone su bienestar.

Con relación a la comparación de las estrategias de afrontamiento por género, las mujeres emplean más las estrategias de buscar apoyo social, esforzarse y tener éxito, preocuparse, buscar pertenencia, hacerse ilusiones, reducción de la tensión, 
autoinculparse, buscar apoyo espiritual y fijarse en lo positivo. En contraparte, los participantes masculinos emplean más estrategias de ignorar el problema, buscar diversiones relajantes y la distracción física.

Jensen (2008) menciona que las mujeres trabajan al lado de su madre desde temprana edad y aprenden a hacerse cargo del cuidado de los demás (hermanos y primos), lo que se vincula a la búsqueda de apoyo social. Por otra parte, este autor señala que los hombres tienen más independencia en la adolescencia, en donde el proceso de alcanzar la hombría suele estar lleno de peligros y conlleva una posibilidad de fracasos; asimismo, el adolescente tiene que demostrar la capacidad de proveer, proteger y procrear. González et al. (2002) refieren que los varones se centran en mejorar sus logros académicos, tienden a utilizar más las estrategias de distracción física, ignorar el problema y lo reservan para sí mismos.

Al respecto, Gaeta y Martín (2009) mencionan que el proceso de afrontamiento puede estar influido por la cultura y contexto (procesos de socialización), por lo que pueden presentarse diferencias en la manifestación de estrategias de afrontamiento ante el estrés.

Tanto para las mujeres como para los hombres, las exigencias suelen intensificarse en la adolescencia y no se toleran muchas desviaciones de las normas. Además, las mujeres y los hombres suelen llevar vidas diferentes, la conducta esperada y el trabajo que desempeñarán de adultos está claramente separada para ambos (Jensen, 2008).

Por otra parte, con relación a la edad, se encontró que los adolescentes de 16 años emplean estrategias de afrontamiento como preocuparse, falta de afrontamiento o no afrontamiento, reducción de la tensión, ignorar el problema, autoinculparse y buscar diversiones relajantes. Ello coincide con Gaeta y Martín (2009), quienes refieren que los chicos de mayor edad son capaces de pensar de manera abstracta, al considerar varios puntos de vista y al evaluar las posibles consecuencias. Asimismo, González et al. (2002) mencionan que debido al surgimiento del pensamiento de las operaciones formales en jóvenes de 15 a 18 años, los de mayor edad poseen un repertorio de incongruencia de afrontamiento más amplio, comparados con los más jóvenes.

La edad supone experiencia y familiaridad con muchas situaciones, lo que conlleva a un mayor conocimiento de los propios recursos, así como generalmente un mejor repertorio de estrategias de afrontamiento. Las estrategias utilizadas por los adolescentes, varían de acuerdo a las fases de la adolescencia y de acuerdo a los cambios cognitivos, así como las demandas ambientales particulares y el apoyo social característicos de los periodos temprano, medio y tardío de la adolescencia (Gaeta y Martín, 2009).

Asimismo, el surgimiento del pensamiento de operaciones formales puede ayudar a los adolescentes a elegir estrategias de afrontamiento efectivas, debido a que pueden ser capaces de pensar de manera abstracta, considerar varios puntos de vista y evaluar las consecuencias. Posteriormente, en el proceso de desarrollo cognitivo, el funcionamiento metacognitivo de autocontrol, el cual se encuentra relacionado con la resolución de problemas y las estrategias de regulación de las emociones, tendrá efectos sobre el afrontamiento adaptativo de los jóvenes (Gaeta y Martín, 2009).

Por tanto, los adolescentes estudiantes de nivel medio superior del Valle de Toluca afrontan el estrés empleando diversas 
estrategias, presentándose diferencias en el género y la edad, siendo estos factores los que pueden intervenir en el empleo de las mismas.

Aunque en el estudio sólo se consideró un rango de edad, de 14 a 17 años, éste contribuye al conocimiento de las estrategias empleadas por adolescentes que se perfilan a un nivel de estudios superiores, es decir, que van a elegir una carrera profesional, lo que lleva al individuo a resolver problemas, conflictos de creencias, valores, limitaciones personales, ambientales.

\section{REFERENCIAS}

Ávila, M., Heredia, M., Gómez, E. y Martínez, L. (2006).Confiabilidad internay estabilidad temporal del cuestionario de sucesos de vida para adolescentes en estudiantes mexicanos. Enseñanza e Investigación en Psicología, 10(11), 97-113.

Bárcenas, A. y Martínez, K. I. (2008). Escala de enfrentamiento para adolescentes: adaptación a población mexicana. México: Universidad Autónoma de Aguascalientes.

Barra, A. E., Cerna, C. R., Kramm, M. D. y Véliz, V. V. (2006). Problemas de Salud, Estrés, Afrontamiento, Depresión y Apoyo Social en Adolescentes. Terapia Psicológica, 1(24), 55-61.

Buendía, J. (1993). Estrés y psicopatología. Madrid: Pirámide.

Cardozo, G. y Alderete, A. M. (2009). Adolescentes en riesgo psicosocial y resiliencia. Psicología desde el Caribe, $23,148-182$.

Casullo, M. (1998). Adolescentes en riesgo. Identificación y orientación psicológica. Buenos Aires: Paidós.

Contreras, G. O., Chávez, B. M. y Aragón, B. L. E. (2012). Pensamiento constructivo y afrontamiento al estrés en estudiantes universitarios. Journal of Behavior, Health \& Social, 1(4), 3953. Recuperado de www.journals. unam.mx/index.php/jbhsi/article/ download/32970/pdf

Enríquez, B. J. F., Lira, M. M. G., Balcázar, N. P., Bonilla, M. M. y Gurrola, P. M. (2009). Adolescentes del siglo XXI. México: Universidad Autónoma del Estado de México.

Frydenberg, E. y Lewis, R. (2000). ACS Escalas de Afrontamiento para Adolescentes. Manual. Madrid: TEA Ediciones.

Gaeta, M. L. y Martín, H. P. (2009). Estrés y adolescencia: Estrategias de afrontamiento y autorregulación en el contexto escolar. Revista de Humanidades, 15, 327-344.

Garrison, K. C. y Garrison, JR. K. C. (1975). Psychology of Adolescence. Englewood Cliffs, NJ: Prentice-Hall.

González, B. R., Montoya, C. I., Martina, C. M. y Bernabéu, V. J. (2002). Relación entre estilos y estrategias de afrontamiento y bienestar psicológico en adolescentes. Psicothema, 2(14), 363-368.

Hernández, S. R., Fernández, C. y Baptista, L. P. (2010). Metodología de la investigación (5ta. ed.). México D.F.: McGraw Hill.

Jensen, A. J. (2008). Adolescencia y adultez emergente. Un enfoque cultural (3ra. ed.). México D.F.: Pearson-Prentice Hall. Lazarus, R. y Folkman, S. (1986). Estrésy procesos cognitivos. Barcelona: Martínez Roca.

Martínez, M. K. I. y Pedroza, C. F. J. (2011). Factores asociados al proceso de recaída en adolescentes consumidores de alcohol. Revista de Psicología, 2(1), 215224. Recuperado de http://infad.eu/ RevistaINFAD/2011/n1/volumen2/ INFAD_010223_215-224.pdf

Moysén, C. A. (2011). Modelo de consumo de alcohol en estudiantes universitarios con respecto a sucesos de vida y estrategias 
de afrontamiento. Tesis doctoral inédita. México D.F.: Universidad Iberoamericana.

Oblitas, G. L. A. y Becoña, E. (2000). Psicología de la salud. México D.F.: Plaza y Valdés.

Solís, M. C. y Vidal, M. A. (2006). Estilos y estrategias de afrontamiento en adolescentes. Revista de psiquiatría y salud mental. 1, 33-39. Recuperado de http://www.hhv.gob.pe/ revista/2006/3\%20ESTILOS\%20 Y\% 20 ESTRATEGIAS \% 20 DE \% 20 AFRONTAMIENTO.pdf

Veloso, B. C., Caqueo, A. W., Caqueo, U. A., Muñoz, S. Z. y Villegas, A. F. (2010). Estrategias de afrontamiento en adolescentes. Fractal: Revista de Psicología. 1(22), 2334.

Wade, C. y Tavris, C. (2003). Psicología (7ma. ed.). Madrid: Pearson.

(c) Los autores. Este artículo es publicado por la Revista Digital de Investigación en Docencia Universitaria del Área de Investigación de la Dirección de Calidad Educativa, Universidad Peruana de Ciencias Aplicadas. Este es un artículo de acceso abierto, distribuido bajo los términos dela Licencia Creative Commons Atribución-CompartirIgual4.0 Internacional.(http://creativecommons.org/licenses/by-sa/4.0/), que permite el uso no comercial, distribución y reproducción en cualquier medio, siempre que la obra original sea debidamente citada. 